\title{
Comparison of a Single Dose Fibrinogen Concentrate with Placebo and Blood Transfusions after Surgery on the Aortic Arch: A Prematurely Ended Randomized Controlled Trial
}

\author{
Eline A Vlot ${ }^{1 *}$, Christian M Hackeng ${ }^{2}$, Uday Sonker ${ }^{3}$, Robin H Heijmen ${ }^{3}$, Eric PA van Dongen ${ }^{1}$ and Peter G Noordzij ${ }^{1}$ \\ ${ }^{1}$ Department of Anesthesia, Intensive Care and Pain Medicine, St Antonius Hospital, Nieuwegein, The Netherlands \\ ${ }^{2}$ Department of Clinical Chemistry, St Antonius Hospital, Nieuwegein, The Netherlands \\ ${ }^{3}$ Department of Cardiac Surgery, St Antonius Hospital, Nieuwegein, The Netherlands
}

${ }^{*}$ Corresponding author: Eline A Vlot, Department of Anesthesia, Intensive Care and Pain Medicine, St Antonius Hospital, Nieuwegein, The Netherlands

Received: March 18, 2021; Accepted: March 25, 2021; Published: October 11, 2021

\begin{abstract}
Background: Aortic replacement surgery is often complicated by significant bleeding due to perioperative coagulopathy. Data regarding the effectiveness of treatment with fibrinogen concentrate to reduce perioperative blood transfusion in aortic replacement surgery in prior studies have shown conflicting results.
\end{abstract}

Methods: A randomized, placebo-controlled, double blind, clinical trial of a single dose fibrinogen concentrate versus placebo for treatment of postcardiopulmonary bypass coagulopathy in patients undergoing elective surgery for thoracic arch aneurysm with deep hypothermic circulatory arrest.

Results: Twenty patients were randomized to fibrinogen concentrate $(\mathrm{N}=10)$ or placebo $(\mathrm{N}=10)$. The recruitment of study patients was prematurely ended due to a low inclusion rate. Five (50\%) patients in the fibrinogen group and two (20\%) patients in the placebo group had at least one perioperative blood transfusion ( $\mathrm{P}=0.196)$. The 5-minute bleeding mass after study medication was $65 \mathrm{~g}$ [39-104] in the fibrinogen group and $61 \mathrm{~g}$ [40-108] in the control group ( $\mathrm{P}=0.910)$. Postoperative blood loss was $450 \mathrm{ml}$ [300-655] in the fibrinogen group and $510 \mathrm{ml}$ [415-650] in the control group ( $\mathrm{P}=0.405)$. After study medication, maximum clot strength and time from initial clot formation until maximum firmness improved significantly in the fibrinogen group but not in the control group.

Conclusions: Due to small volumes of postoperative blood loss and premature study termination, a beneficial effect of fibrinogen concentrate on the number of blood transfusions after aortic replacement surgery could not be established. However, treatment with fibrinogen effectively restored the postoperative fibrinogen concentration to preoperative levels and increased maximum clot strength after cardiopulmonary bypass.

Keywords: Hemostasis, Fibrinogen, Point of care systems, Thoracic aorta surgery

\section{Introduction}

Aortic replacement surgery is often complicated by significant bleeding due to perioperative coagulopathy. Consumption and dilution of clotting factors, inflammation and fibrinolysis, Hypothermic Circulatory Arrest (HCA) and long Cardiopulmonary Bypass (CPB) time increase the need for blood transfusions to treat perioperative blood loss [1]. Although blood transfusion is increasingly safe, evidence suggests that it remains associated with adverse clinical outcomes [2]. To reduce the risk of transfusion associated complications, perioperative coagulopathy can be treated with coagulation factor replacement therapies, but adequate powered randomized studies on the efficacy and safety of such therapies are scarce $[3,4]$.

Plasma fibrinogen plays an important role in perioperative hemostasis, but is often reduced to low concentrations during cardiac surgery [5]. Low plasma fibrinogen levels reduce clot firmness and have been associated with bleeding complications [6]. Plasma fibrinogen initiates clot formation and enhances platelet aggregation by binding platelet glycoprotein IIb/IIIa receptors found on platelets $[7,8]$. To prevent depleted plasma fibrinogen levels, cardiac surgery patients can be substituted by plasma transfusion, Fibrinogen Concentrate (FC) or cryoprecipitate. Transfusion with plasma immediately supplies all essential coagulant factors, but requires large volumes to restore plasma fibrinogen levels, with risk of circulatory overload.

Data regarding the effectiveness of treatment with FC to reduce perioperative blood transfusion in aortic replacement surgery in prior studies have shown conflicting results. A landmark randomized trial in 2013 showed that blood transfusion was significantly reduced by FC compared to placebo [9]. However, a follow up multicenter study 
in 2016 could not confirm these results [10]. Fibrinogen concentrate did not reduce blood loss in studies that included a cardiac surgery population with lower bleeding risk (i.e. $<20 \%$ of included patients had aortic replacement surgery) [11]. We therefore conducted a double blind randomized controlled trial in patients with aortic arch replacement surgery with HCA to investigate if intraoperative FC administration reduced the need for allogeneic perioperative blood transfusion within $24 \mathrm{~h}$ after surgery.

\section{Patients and Methods}

\section{Study Design}

A randomized, placebo-controlled, double blind, clinical trial of FC (Hemocomplettan P, CSL Behring, Marburg) versus placebo for treatment of post $\mathrm{CPB}$ coagulopathy in patients undergoing elective aorta replacement surgery with HCA for thoracic aneurysm at a large tertiary hospital for cardiac surgery. Ethical approval was provided by the local ethics committee (Medical Ethics Research Committee United, no. NL45370.020.13) on July $16^{\text {th }} 2014$. The study protocol was registered at the US National Library of Medicine (NCT02299947) and performed in accordance with the Declaration of Helsinki. All patients gave written informed consent prior to entering the study. On July 2015 the protocol was amended after a serious adverse event (one patient died of mesenteric ischemia) and a history of severe atherosclerosis or type $\mathrm{B}$ aortic dissection was added to the exclusion criteria.

\section{Study Population}

Adults with thoracic aneurysm scheduled for aortic replacement surgery were eligible for study participation. Patients with the following medical conditions were not recruited: prior thrombosis or myocardial infarction, congenital coagulation disorder, use of antiplatelet therapy or vitamin $\mathrm{K}$ antagonists within 5 days preceding surgery, a history of severe atherosclerosis or type $\mathrm{B}$ aortic dissection, prior thoracic surgery, pregnancy and pre-operative fibrinogen concentration $<1 \mathrm{~g} / \mathrm{L}$. Patients were informed by telephone at least one week prior to the planned surgical procedure and with their permission written study information was sent by mail. Informed consent was retrieved by a trained research professional one day before surgery. Inclusion took place between August 2014 and July 2018.

\section{Data Collection and Study Procedures}

Patient characteristics were collected from routine preoperative anesthesia assessment and consisted of medical history, current drug use, laboratory tests and echocardiography results. Surgical characteristics and study data were prospectively collected and started at time of hospital admission. Anesthesia and Intensive Care Unit (ICU) management were conducted following local standard operating procedures for cardiac surgery. For Cardiopulmonary Bypass (CPB), non-pulsatile perfusion was used with a flow of 2.0 to $2.41 / \mathrm{min} / \mathrm{m} 2$ and unfractionated heparin was used to target the kaolin Activated Clotting Time (ACT) $>400$ s. After aortic cross-clamping, cardiac arrest was initiated using a cold crystalloid cardioplegia solution (St. Thomas cardioplegia, Pharmacy 'Haagse Ziekenhuizen', The Hague, The Netherlands). Deep HCA with a core temperature of $25^{\circ} \mathrm{C}$ and bilateral antegrade, selective, cerebral perfusion preserved organ function during aortic replacement surgery. Patients were weaned from $\mathrm{CPB}$ after rewarming (temperature $>35.5^{\circ} \mathrm{C}$ ). Heparin was reversed with protamine sulfate $(0.75 \mathrm{mg}$ per $100 \mathrm{U}$ of heparin) and tranexamic acid was administered to each patient.

\section{Blood Product Transfusion Algorithm}

Blood product transfusion was performed according to a local transfusion protocol. The trigger for Red Blood Cell (RBC) transfusion was a Hematocrit $(\mathrm{Ht})<0.20$ during $\mathrm{CPB}$ or $<0.25$ after $\mathrm{CPB}$; a Hemoglobin $(\mathrm{Hb})<4.4 \mathrm{mmol} / 1(7.1 \mathrm{~g} / \mathrm{dl})$ during ICU stay. Plasma transfusion was based on intraoperative blood loss (i.e. the number of transfused cell saver units or clinical signs of coagulopathy after protamine administration) or ongoing blood loss and an international normalized ratio of prothrombin time (INR) $>1.5$ after ICU arrival. Platelet transfusion depended on clinical signs of coagulopathy and/ or low Platelet Count (PC) $<100 \times 10^{9} / 1$.

\section{Blood Sampling and Coagulation Tests}

Blood samples were collected from an arterial line at five perioperative time points: T1. At baseline after induction of anesthesia; T2. During CPB after initiation of rewarming; T3. Post-CPB, after protamine administration; T4. Post-CPB, after treatment with study medication and T5. At ICU arrival. Whole blood was sampled in K2EDTA and 3.2\% sodium citrate tubes (BD Vacutainer) for conventional blood tests and coagulation assays at the hospital laboratory ( $\mathrm{Hb}, \mathrm{Ht}, \mathrm{PC}$, Fibrinogen (Clauss assay, STA-R Evolution analyzer, Diagnostica STAGO, France), activated partial thromboplastin time (aPTT)). Viscoelastic Point-of-Care (POC) testing was performed on a TEG 5000-analyzer (Haemonetics Corp., USA). Whole blood POC tests were performed at the operation room complex by a research professional within 5 minutes after sampling. Viscoelastic POC tests included: kaolin initiated clotting time (R; min.), time from initial clot formation until maximum firmness (Alpha Angle (AA); degrees), maximum clot strength (MA; mm) and Functional Fibrinogen (FF).

\section{Intervention}

Study medication was prepared by a trial pharmacist after aortic arch reconstruction during $\mathrm{CPB}$ rewarming. Fibrinogen Concentrate (FC) was dissolved in a blinded infusion bag with sterile water (1 gram per $50 \mathrm{ml}$ ). Fibrinogen dose was based on patient weight, using the following dosing regimen: $4 \mathrm{~g} / 200 \mathrm{ml}$ for weight $<70 \mathrm{~kg}$ $=; 6 \mathrm{~g} / 300 \mathrm{ml}$ for weight $70-90 \mathrm{~kg} ; 8 \mathrm{~g} / 400 \mathrm{ml}$ for weight $>90 \mathrm{~kg}$ ) [12]. Placebo was a weight adjusted equivalent volume of sodium chloride $0.9 \%$ (Freeflex, Fresenius Medical Care Nederland B.V). All study medication was administered through an 18-gauche peripheral coloured intravenous line.

Study medication was only administered if clinically relevant bleeding occurred after $\mathrm{CPB}$ and when completion of surgical hemostasis for focal bleeding was accomplished by the surgeon. The 5-minute intraoperative bleeding mass was determined by weighing dry surgical gauzes, applying them into the surgical field for 5 minutes with no touch or irrigation and weighing them again. Blood volume 
Eline A Vlot (2021) Comparison of a Single Dose Fibrinogen Concentrate with Placebo and Blood Transfusions after Surgery on the Aortic Arch:

A Prematurely Ended Randomized Controlled Trial

weight between 60 and 250 grams was classified as clinically relevant coagulopathic bleeding [9]. After administration of study medication, 5-minute bleeding mass was measured again.

\section{Outcomes}

The primary outcome parameter was number of perioperative transfused allogeneic blood products within $24 \mathrm{~h}$ after surgery. Secondary outcome parameters were blood loss after surgery (chest tube drainage volume $24 \mathrm{~h}$ after surgery), re-operation (30 days) and postoperative mortality (30 days). Tertiary outcome parameters included the course of perioperative plasma fibrinogen levels and viscoelastic POC coagulation parameters.

\section{Randomization and Blinding}

Before surgery patients were randomly allocated by a clinical pharmacist to receive $\mathrm{FC}$ or placebo using block randomization (eight patients per block). Blinding of the investigators and surgical team was ensured by maintaining equivalent volumes of study medication in an infusion bag wrapped in aluminum foil.

\section{Sample Size}

Based on historical transfusion data in patients with aortic replacement surgery from our institution (median number of 24 h blood transfusions $9 \pm 7$ ) we hypothesized that two groups of 39 patients were required to demonstrate a $50 \%$ reduction of blood transfusion products in $60 \%$ of patients.

\section{Statistical Analysis}

Descriptive statistics were calculated for all parameters. Categorical variables were described as numbers and percentages. Continuous data were described as mean (standard deviation) and median [interquartile range] for normally and non-normally distributed data. Normality was tested using visual inspection of histograms. To compare categorical variables between groups Pearson chi-square test or, in case of small sample sizes, the non-parametric Fisher's exact test was used. Statistical comparison of continuous variables was performed using the Student's t-test for normally distributed data and the Mann-Whitney U test for non-normally distributed data. The sign test was applied for directional changes in paired coagulation parameters before and after administration of study medication. For estimating a difference in the primary outcome parameter between FC group and control group, Mann-Whitney U test was conducted on the number of transfused allogeneic blood products according to study medication treatment. Exploratory analysis was performed for secondary endpoints postoperative $24 \mathrm{~h}$ blood loss, re-operation and postoperative mortality. There were no missing data for the primary outcome. Missing data for secondary and tertiary outcome parameters were assumed missing completely at random and were excluded from analysis. No adjustment for multiple statistical comparisons was used.

\section{Results}

\section{Study Population}

Between August 2014 and July 2018, 171 patients with a thoracic aneurysm were screened for study participation and 73 patients were considered eligible and received study information (Figure 1). Twentyseven patients provided informed consent and were randomized to receive FC or placebo. During surgery two patients were excluded because aortic replacement surgery was performed without HCA and five patients had insufficient intraoperative bleeding. On November 2014 the data and safety monitoring board was consulted early for a serious adverse event (one patient died of mesenteric ischemia) and concluded that the study should be continued based on the results of an interim analysis. The recruitment of study patients was terminated in December 2018 due to a low inclusion rate.

Twenty patients were treated with $\mathrm{FC}(\mathrm{N}=10)$ or placebo $(\mathrm{N}=10)$. Mean age was $63( \pm 12)$ years, mean weight was $87( \pm 20) \mathrm{kg}$ and six $(30 \%)$ patients were female. Baseline characteristics are listed in Table 1. Fibrinogen concentration at the end of CPB and before study medication was $1.5[1.4-1.7] \mathrm{g} / \mathrm{l}$ for the fibrinogen group and 1.6 [1.4-2.1] g/l for the control group (supplementary Table 1 ). The mean decrease in plasma fibrinogen concentration compared to baseline was $47 \%( \pm 7)$ and $18(90 \%)$ patients had a fibrinogen concentration $<2$

\begin{tabular}{|c|c|c|c|}
\hline Demographics & Fibrinogen $n=10$ & Placebo $n=10$ & Missing \\
\hline Age, median [IQR], y] & $67[55-74]$ & $64[50-67]$ & 0 \\
\hline Female, No (\%) & $4(40)$ & $2(20)$ & 0 \\
\hline Weight, mean (SD), kg & $86(24)$ & $89(15)$ & 0 \\
\hline EuroSCORE, median [IQR] & $7.5[5-10]$ & $6.5[5-8]$ & 0 \\
\hline Diabetes, No (\%) & $0(0)$ & $0(0)$ & 0 \\
\hline Hypertension, No (\%) & $6(60)$ & $6(60)$ & 0 \\
\hline $\begin{array}{r}\text { LVEF \%, No (\%) } \\
<30 \\
30-50 \\
>50\end{array}$ & $\begin{array}{c}0 \\
3(30) \\
7(30)\end{array}$ & $\begin{array}{c}0 \\
3(30) \\
7(70)\end{array}$ & 0 \\
\hline Aortic Valve surgery, No (\%) & $4(40)$ & $8(80)$ & 0 \\
\hline $\mathrm{CPB}$ time, median [IQR], $\min$ & 198 [174-225] & $222[195-259]$ & 0 \\
\hline Cerebral perfusion time, median [IQR], min & $30[26-47]$ & 33 [27-57] & 0 \\
\hline $\begin{array}{r}\text { Transfusion autologous blood, median } \\
{[\mathrm{IQR}], \mathrm{ml}}\end{array}$ & $833[600-1700]$ & $\begin{array}{c}1100[550- \\
1300]\end{array}$ & 1 \\
\hline $\begin{array}{r}\text { 5-min bleeding mass before } \mathrm{SM} \text {, median } \\
{[\mathrm{IQR}], \mathrm{ml}}\end{array}$ & $131[90-188]$ & $142[79-158]$ & 0 \\
\hline $\begin{array}{r}\text { 5-min bleeding mass after SM, median } \\
{[\mathrm{IQR}], \mathrm{ml}}\end{array}$ & 65 [39-104] & $61[40-108]$ & 0 \\
\hline \multicolumn{4}{|l|}{ Laboratory } \\
\hline $\mathrm{Hb}, \mathrm{mmol} / \mathrm{l}$ & $8.0[7.3-8.7]$ & $7.8[6.7-8.9]$ & 0 \\
\hline $\mathrm{Ht}, \%$ & $0.38[0.35-0.40]$ & $0.37[0.33-0.41]$ & 0 \\
\hline Platelet count $\times 10^{9} / 1$ & 191 [163-212] & 208 [161-249] & 0 \\
\hline Fibrinogen $\mathrm{g} / \mathrm{l}$ & $3.1[2.7-3.2]$ & $3.1[2.7-4.0]$ & 0 \\
\hline Aptt, s & 34.9 [32.3-36.9] & 34.4 [31.9-38.7] & 3 \\
\hline \multicolumn{4}{|l|}{ Viscoelastic POC } \\
\hline TEG-MA, mm & $67.4[65.2-69.8]$ & $68.5[64.6-72.7]$ & 0 \\
\hline TEG-R, min & $7.2[4.7-8.9]$ & $6.9[3.9-8.6]$ & 0 \\
\hline TEG-AA, ${ }^{\circ}$ & $62.1[59.7-68.3]$ & $65.8[63.4-71.8]$ & 0 \\
\hline FF-MA, mm & $26.7[22.5-40.6]$ & $30.9[27.5-38.7]$ & 1 \\
\hline
\end{tabular}

CPB: Cardiopulmonary Bypass, SM: Study Medication, TEG: Thromboelastography, R: Kaolin initiated Clotting Time, AA: Angle from Initial Clot Formation Until Maximum Firmness, MA: Maximum Clot Strength, FF: Functional Fibrinogen. 


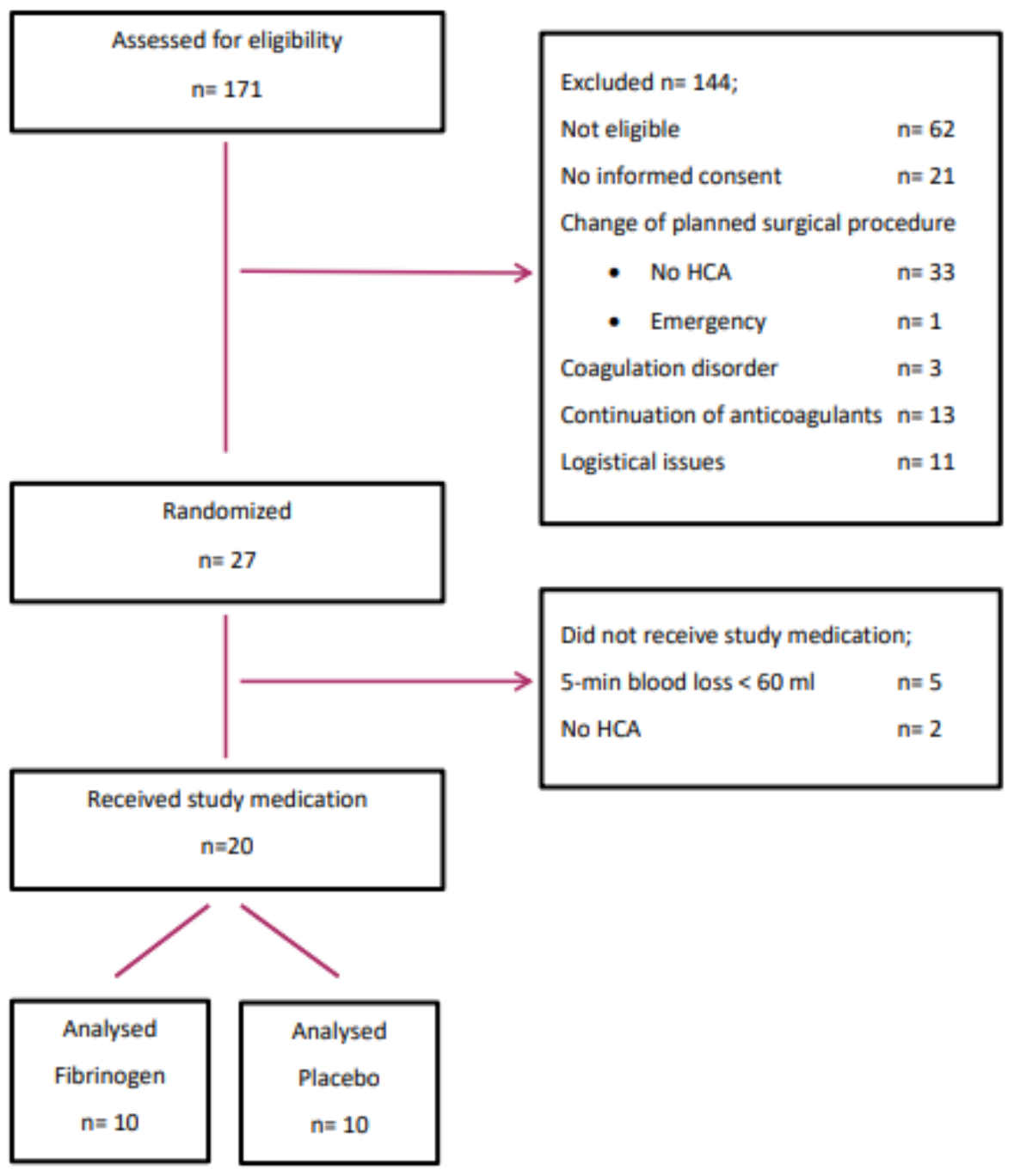

Legends; HCA; hypothermic circulatory arrest.

Figure 1: Patient flow diagram.

Table 2: Perioperative blood transfusions.

\begin{tabular}{|l|c|c|c|c|}
\hline & Fibrinogen & Placebo & P & Missing \\
\hline Intraoperative blood transfusions, units (range) & & & & \\
\hline RBC & $3(1-2)$ & $1(1)$ & 0.503 & 0 \\
\hline Plasma & $1(1)$ & $2(2)$ & 0.942 & 0 \\
\hline Platelets & $2(1)$ & $1(1)$ & 0.542 & 0 \\
\hline Postoperative blood transfusions (24 h), units (range) & & & & \\
\hline RBC & $1(1)$ & 0 & 0.317 & 0 \\
\hline Plasma & $2(2)$ & 0 & 0.317 & 0 \\
\hline Platelets & $1(1)$ & 0 & 0.317 & 0 \\
\hline
\end{tabular}

RBC: Red Blood Cells.

$\mathrm{g} / 1$. After FC administration the plasma concentration increased from 1.5 [1.4-1.7] g/l to 3.1 [2.8-3.3] g/l $(\mathrm{P}=0.005)$. At time of ICU arrival fibrinogen concentration was $3.0[2.6-3.3] \mathrm{g} / \mathrm{l}$ in the fibrinogen group and $1.8[1.5-2.2] \mathrm{g} / 1$ in the control group $(\mathrm{P}=0.001)$. The difference in plasma fibrinogen concentration was no longer present at $24 \mathrm{~h}$ after surgery (4.4 [3.4-4.7] g/l for the fibrinogen group and 3.6 [3.2-4.4] g/1 for the control group, $\mathrm{P}=0.267)$.

\section{Blood Transfusion and Blood Loss}

Five $(50 \%)$ patients in the fibrinogen group and two $(20.0 \%)$ patients in the placebo group had at least one perioperative blood transfusion $(\mathrm{P}=0.196)$. Number and types of perioperative blood transfusions are presented in Table 2. Intraoperative bleeding after CPB was not different between both groups (5-minute bleeding mass 131 [90-188] g for the fibrinogen group and 142 [79-158] g for the control group, $\mathrm{P}=0.821$ ). After study medication 5-minute bleeding mass was reduced by $52 \%$ [33-67] in patients that received FC compared to $32 \%$ [16-80] in patients treated with placebo $(\mathrm{P}=0.705)$. Median postoperative blood loss was $490 \mathrm{ml}$ [393-635]. Postoperative blood loss was $450 \mathrm{ml}$ [300-655] for the fibrinogen group and $510 \mathrm{ml}$ [415-650] for the control group $(\mathrm{P}=0.405)$.

One patient in the fibrinogen group had a reoperation because of mesenteric arterial occlusion. In both groups one patient died during hospital stay, cause of death was mesenteric ischemia in the fibrinogen patient and sepsis in the placebo patient. One patient in the placebo group suffered from a non-fatal stroke (Figure 2). 


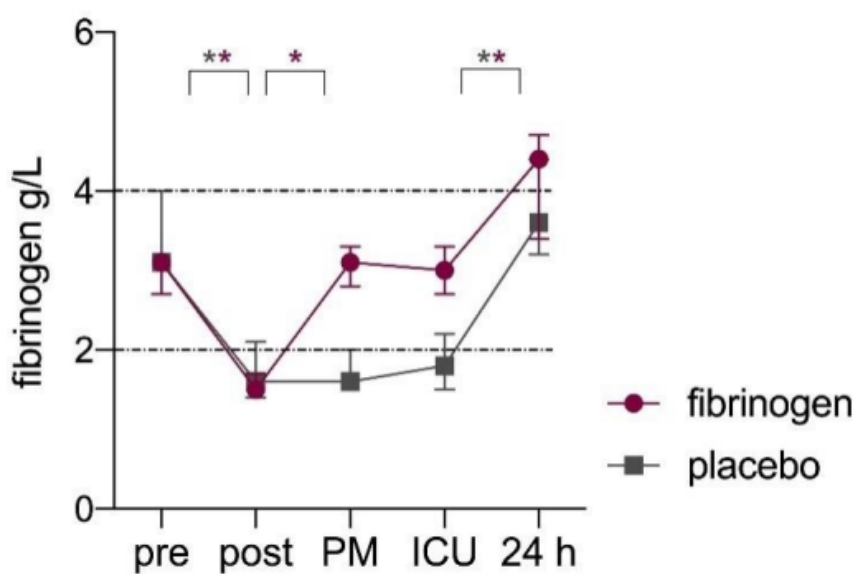

Figure 2: Perioperative fibrinogen concentrations in fibrinogen (purple) and placebo (grey) group.

Legends; Median values and error bars, IQR. Dashed lines indicate reference lines. Pre; baseline, post; post CPB, PM; post study medication. ${ }^{\star} \mathrm{P}<0.05$.

\section{TEG R}

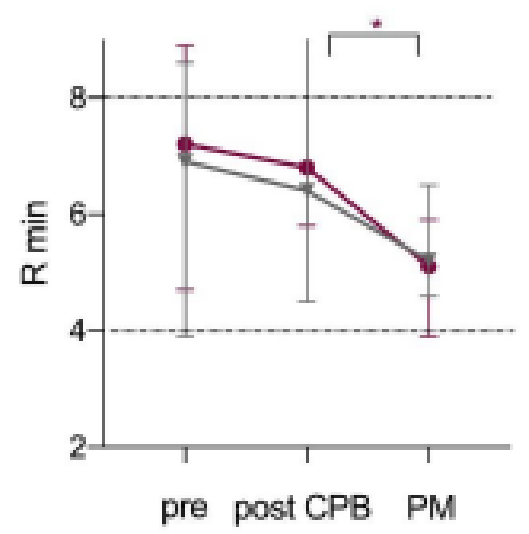

TEG MA
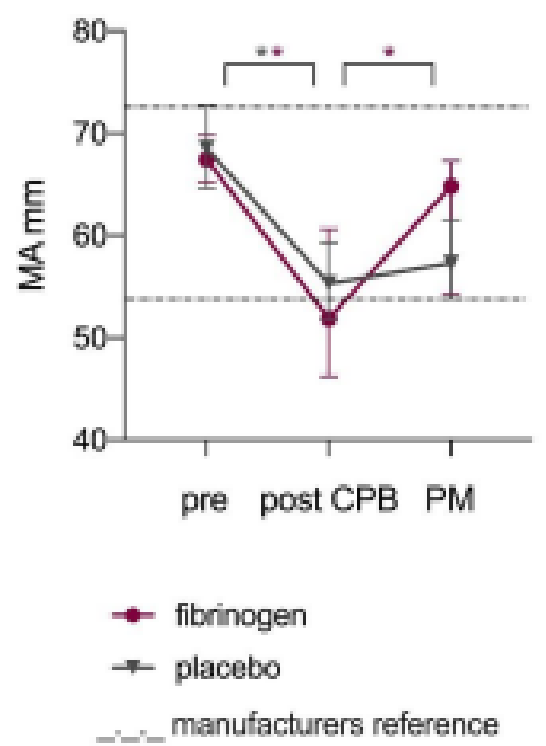

\section{Viscoelastic POC Tests}

Perioperative viscoelastic POC test results are presented in Figure 3. Values at baseline, post-CPB and after study medication were similar between both groups (Supplementary Table 2). A reduction in maximum clot strength between baseline and post-CPB ( $\triangle$ TEG-MA) was related to intraoperative blood loss $(\mathrm{r}=-0.468$ for 5 -min bleeding mass, $\mathrm{P}=0.043$ ). After study medication maximum clot strength and time from initial clot formation until maximum firmness improved significantly in the FC group but not in the control group (Figure $3)$. Test results for maximum clot strength and functional fibrinogen returned to baseline in the FC group (-7\% difference compared to baseline for TEG-MA $(\mathrm{P}=0.203)$ and $-15 \%$ difference for FF-MA $(\mathrm{P}=0.241))$ but did not in the control group $(-16 \%$ difference compared to baseline for TEG-MA $(\mathrm{P}=0.005)$ and $-53 \%$ difference for FF-MA $(\mathrm{P}=0.008)$ ).
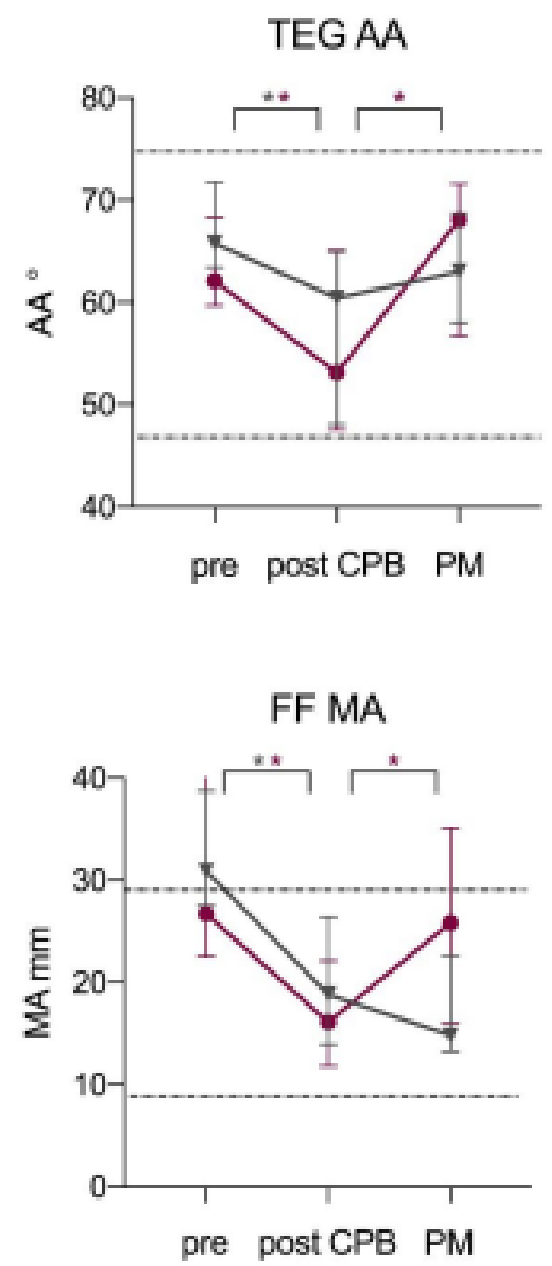

Figure 3: Perioperative viscoelastic POC test results.

Legends; Data markers indicate median and error bars, IQR. Dashed lines indicate manufacturers reference lines. Pre; baseline, $\mathrm{PM}$; post study medication. ${ }^{\star} \mathrm{P}<0.05$. 


\section{Discussion}

This study aimed to determine the effect of intraoperative treatment with FC on perioperative blood product transfusion in patients with elective aortic arch replacement surgery with HCA. A difference in number of blood transfusions after treatment with FC could not be demonstrated because the recruitment of study patients was prematurely ended due to a low inclusion rate and sample size was insufficient to answer our primary research question. Patients treated with FC showed an improvement in fibrinogen concentration, maximum clot strength and time from initial clot formation until maximum firmness directly after $\mathrm{CPB}$, compared to patients treated with placebo.

Prior studies on the effect of treatment with FC to reduce blood transfusions in cardiac surgery patients have shown conflicting results [9-11,13-15]. A single-center randomized trial published in 2013, demonstrated that intra-operative treatment with FC resulted in an $85 \%$ reduction of blood transfusions in patients undergoing aortic replacement surgery [9]. Total avoidance of transfusion was achieved in almost half of the FC patients compared to none of the placebo patients. However, the number of blood transfusions in the control group was very high (median 13 units vs. 2 units in the FC group) and the favorable effect of FC treatment on blood transfusion after aortic replacement surgery could not be confirmed in a follow up multi-center study in 2016 with a similar design [10]. In that study, the number of transfusions in the placebo group declined to a median of 3 units [0-7] and was even lower than the number of transfusions in the FC group (median 5 units) [2-11]. A clear explanation for the higher transfusion rate in the FC group was not found, but could have been the result of poor protocol adherence [16]. The large decline in perioperative blood transfusions over time in patients undergoing aortic replacement surgery, irrespective of FC treatment, was also witnessed in our institution. Our historical transfusion data that were used for sample size analysis in 2014, showed a much higher number of transfusions than the control group of our randomized trial (median $9 \pm 7$ vs. median $0 \pm 0$ ). Therefore, it seems unlikely that our sample size would have been sufficient to demonstrated a significant difference in transfusions between both groups, if patient recruitment had not been prematurely ended. The implementation of a patient blood management program in 2015 that consisted of lower transfusion thresholds, an intraoperative POC transfusion algorithm, modified surgical and CPB techniques to reduce blood loss and limit intraoperative anemia could explain the overall reduction of transfusions over time in patients undergoing aortic replacement surgery in our institution.

Our results showed that treatment with $\mathrm{FC}$ resulted in an improvement of maximum clot strength and time from initial clot formation until maximum firmness compared to placebo. This finding is relevant as a reduction in maximum clot strength between baseline and post-CPB was correlated to intraoperative blood loss. In surgical patients, viscoelastic coagulation tests are used for the early diagnosis of coagulation disorders, to guide transfusion management and consequently reduce postoperative bleeding and blood product consumption [17-19]. In cardiac surgery patients monitoring of fibrinogen function with viscoelastic tests showed a better clinical performance than routine coagulation tests as a standardized, more reliable and valid laboratory tool for monitoring of the fibrinogen contribution to the clot formation [3]. Maximum clot strength was found to be the best viscoelastic predictor of postoperative blood loss, while none of the routine coagulation tests showed any correlation with postoperative bleeding [19,20].

Fibrinogen plays a key role in hemostasis and a negative association exists between plasma fibrinogen levels and blood loss after cardiac surgery [8,21-23]. Studies have shown that patients with normal or elevated fibrinogen levels experience fewer bleeding complications than patients with low fibrinogen levels $[23,24]$. In post-CPB hemostasis, fibrin formation is significantly more deteriorated than the platelet component of whole blood clot strength, suggesting that initial management of coagulopathy following cardiac surgery should focus on improving fibrin formation [25]. While the critical level of plasma fibrinogen, in relation to perioperative blood loss, remains subject of debate, there are experimental and clinical data describing that fibrinogen improves clot strength dose dependently [26,27]. In our study the median fibrinogen concentration after CPB was below the reference range in $90 \%$ of patients but most patients had little blood loss and a beneficial effect of FC treatment to reduce the number of blood transfusion products could not be established. However, we were able to demonstrate that FC treatment effectively restored fibrinogen levels and maximum clot strength to preoperative levels. Considering the relationship between maximum clot strength and bleeding, viscoelastic testing can guide the physician in the primary replacement of fibrinogen to reduce postoperative blood loss.

\section{Limitations}

Our trial was prematurely ended due to low inclusion rates. More than expected, patients were not eligible for study participation due to use of anticoagulants or a history of thrombosis. Also, we overestimated the willingness of high-risk cardiac surgery patients to participate in a clinical intervention study. The main reasons to refrain from study participation were anxiety for surgery and fear for adverse outcome. We aimed to include patients at high risk for postoperative bleeding based on type of surgery, use of HCA and historical data. However, overall blood loss was low. This may be the result of a Hawthorne effect. Also, different aspects of a patient blood management program could have influenced blood loss in favor of patient outcome.

\section{Conclusion}

This study demonstrated that low fibrinogen levels are common after elective aortic replacement surgery with HCA. Treatment with FC effectively restored fibrinogen levels, improved maximum clot strength and time until maximum firmness but a reduction in blood loss compared to patients treated with placebo could not be demonstrated. 
Eline A Vlot (2021) Comparison of a Single Dose Fibrinogen Concentrate with Placebo and Blood Transfusions after Surgery on the Aortic Arch: A Prematurely Ended Randomized Controlled Trial

\section{References}

1. Görlinger K, Shore-Lesserson L, Dirkmann D, Hanke A, Rahe-Meier N, et al. (2013) Management of Hemorrhage in Cardiothoracic Surgery. J Cardiothorac Vasc Anesth 27: S20-34. [crossref]

2. Task Force on Patient Blood Management for Adult Cardiac Surgery of the European Association for Cardio-Thoracic Surgery (EACTS) and the European Association of Cardiothoracic Anaesthesiology (EACTA), Boer C, Meesters MI, Milojevic M, Benedetto U, Bolliger D, Heymann C, et al. 2017 EACTS/EACTA Guidelines on patient blood management for adult cardiac surgery. J Cardiothorac Vasc Anesth. 2018; 32: 88-120.

3. Erdoes G, Koster A, Meesters MI, Ortmann E, Bolliger D, et al. (2019) The Role of Fibrinogen and Fibrinogen Concentrate in Cardiac Surgery: An International Consensus Statement from the Haemostasis and Transfusion Scientific Subcommittee of the European Association of Cardiothoracic Anaesthesiology. Anaesthesia 74: 1589-1600. [crossref]

4. Fitzgerald J, Lenihan M, Callum J, Mc Cluskey SA, Srinivas C, et al. (2018) Use of prothrombin complex concentrate for management of coagulopathy after cardiac surgery: a propensity score matched comparison to plasma. Br J Anaesth 120: 928934. [crossref]

5. Karkouti K, Callum J, Crowther MA , McCluskey SA, Pendergrast J, et al. (2013) The Relationship Between Fibrinogen Levels After Cardiopulmonary Bypass and Large Volume Red Cell Transfusion in Cardiac Surgery. Anesth \& Analg 117: 14-22. [crossref]

6. Essa Y, Zeynalov N, Sandhaus T, Hofmann M, Lehmann T, et al. (2018) Low Fibrinogen Is Associated with Increased Bleeding-Related Re-exploration after Cardiac Surgery. Thorac Cardiovasc Surg 66: 622-628. [crossref]

7. Levy JH, Goodnough LT (2015) How I use fibrinogen replacement therapy in acquired bleeding. Blood 125: 1387-1393. [crossref]

8. Levy JH, Welsby, Goodnough LT (2014) Fibrinogen as a therapeutic target for bleeding: a review of critical levels and replacement therapy. Transfusion 54: 13891405. [crossref]

9. Rahe-Meyer N, Solomon C, Hanke A, Schmidt DS, Knoerzer D, et al. (2013) Effects of fibrinogen concentrate as first-line therapy during major aortic replacement surgery: a randomized, placebo-controlled trial. Anesthesiology 118: 40-50. [crossref]

10. Rahe-Meyer N, Levy JH, Mazer CD, Schramko A, Klein AA, et al. (2016) Randomized evaluation of fibrinogen vs placebo in complex cardiovascular surgery (REPLACE) a double-blind phase III study of haemostatic therapy. Br J Anaesth 117: 41-51. [crossref]

11. Bilecen S, de Groot JA, Kalkman CJ, Spanjersberg AJ, Bruinsma GJBB, et al. (2017) Effect of Fibrinogen Concentrate on Intraoperative Blood Loss among Patients With Intraoperative Bleeding During High-Risk Cardiac Surgery: A Randomized Clinical Trial. JAMA 317: 738-747. [crossref]

12. Collins PW, Solomon C, Sutor K, Crispin D, Hochleitner G, et al. (2014) Theoretical modelling of fibrinogen supplementation with therapeutic plasma, cryoprecipitate, or fibrinogen concentrate. Br J Anaesth 113: 585-595. [crossref]

13. Ranucci M, Baryshnikova E, Crapelli GB, Rahe-Meier N, Menicanti L, et al. (2015) Randomized, double-blinded, placebo-controlled trial of fibrinogen concentrate supplementation after complex cardiac surgery. JAHA 4: e002066. [crossref]
14. Jeppsson A, Waldén K, Roman-Emanuel C, Thimour-Bergstrom L, Karlsson M (2016) Preoperative supplementation with fibrinogen concentrate in cardiac surgery: A randomized controlled study. Br J Anaesth 116: 208-214. [crossref]

15. Lee SH, Lee SM, Kim CS, Cho HS, Lee JH, et al. (2014) Fibrinogen recovery and changes in fibrin-based clot firmness after cryoprecipitate administration in patients undergoing aortic surgery involving deep hypothermic circulatory arrest. Transfusion 54: 1379-1387. [crossref]

16. Rahe-Meyer N, Levy JH, Mazer CD, Schramko A, Klein AA, et al. (2019) Randomized evaluation of fibrinogen versus placebo in complex cardiovascular surgery: post hoc analysis and interpretation of phase III results. Interact Cardiovasc Thorac Surg 28: 566-574. [crossref]

17. Serraino GF, Murphy GJ (2017) Routine use of viscoelastic blood tests for diagnosis and treatment of coagulopathic bleeding in cardiac surgery: updated systematic review and meta-analysis. Br J Anaesth 118: 823-833. [crossref]

18. Meco M, Montisci A, Giustiniano E, Greco M, Pappalardo F, et al. (2020) Viscoelastic Blood Tests Use in Adult Cardiac Surgery: Meta-Analysis, Meta-Regression, and Trial Sequential Analysis. J Cardiothorac Vasc Anesth 34: 119-127. [crossref]

19. Welsby IJ, Jiao K, Ortel TL, Brudney CS, Roche AM, et al. (2006) The kaolin-activated Thrombelastograph predicts bleeding after cardiac surgery. J Cardiothorac Vasc Anesth 20: 531-535. [crossref]

20. Sharma S, Kumar S, Tewari P, S Pande, Murari M (2018) Utility of thromboelastography versus routine coagulation tests for assessment of hypocoagulable state in patients undergoing cardiac bypass surgery. Ann Card Anaesth 21: 151-157. [crossref]

21. Levy JH, Szlam FAHA, Tanaka KA, Sniesienski RM (2012) Fibrinogen and Hemostasis: A Primary Hemostatic Target for the Management of Acquired Bleeding. Anesth \& Analg 114: 261-274. [crossref]

22. Gielen C, Dekkers O, Stijnen T, Brand A, Klautz R, et al. (2014) The effects of preand postoperative fibrinogen levels on blood loss after cardiac surgery: a systematic review and meta-analysis. Interact Cardiovasc Thorac Surg 18: 292-298. [crossref]

23. Kawashima S, Suzuki Y, Sato T, Kikura M, Katoh T, et al. (2016) Four-Group Classification Based on Fibrinogen Level and Fibrin Polymerization Associated With Postoperative Bleeding in Cardiac Surgery. Clin Appl Thromb Hemost 22: 648-655. [crossref]

24. Blome M, Isgro F, Kiessling AH, Skuras J, Haubelt H, et al. (2005) Relationship between factor XIII activity, fibrinogen, haemostasis screening tests and postoperative bleeding in cardiopulmonary bypass surgery. Thromb Haemost 93: 1101-1107. [crossref]

25. Solomon C, Rahe-Meyer N, Sørensen B (2011) Fibrin formation is more impaired than thrombin generation and platelets immediately following cardiac surgery. Thromb Res 128: 277-282. [crossref]

26. Velik-Salchner C, Haas T, Innerhofer P, Streif W, Nussbaumer W, et al. (2007) The effect of fibrinogen concentrates on thrombocytopenia. J Thromb Haemost 5: 10191025. [crossref]

27. Schenk B, Lindner AK, Treichl B, Bachler M, Hermann M, et al. (2016) Fibrinogen supplementation ex vivo increases clot firmness comparable to platelet transfusion in thrombocytopenia. Br J Anaesth 117: 576-582. [crossref]

\section{Citation:}

Vlot EA, Hackeng CM, Sonker U, Heijmen RH, van Dongen EPA, et al. (2021) Comparison of a Single Dose Fibrinogen Concentrate with Placebo and Blood Transfusions after Surgery on the Aortic Arch: A Prematurely Ended Randomized Controlled Trial. Integr J Anesth Sur Volume 2(1): 1-7. 